\title{
A Study on ENC Application of Line Generalization Method
}

\author{
† Hyun-Joo Ko, Se-Woong Oh*, Sang-Hyun Suh**, Woo-Sung Shim***, Chung Yoon***** \\ $\dagger, *, * *, * * *$ Korea Ocean Research \& Development Institute, Daejeon 305-343, Korea \\ ****Chung-nam National University
}

\begin{abstract}
N a v i g a t i o n ~ s t r a t e g y ~ I M O ~ p r o m o t e s ~ i s ~ d e f i n e d ~ a s ~ i t ~ i s ~ n e c e s s a r y ~ f o r ~ n e t w o r k ~ a n d ~ i n f o r m a t i o n ~ s t a n d a r d ~ t o ~ p r o v i d e ~ v a r i o u s ~}$ maritime safety informations to in land and on board users, and it is expected to provide a large amount and diverse kinds of maritime spatial information services to them. However, as there are some limits to transmit that by current mobile maritime communication technologies, it is required to simplify and optimize the information. In this study, tree node method is applied to ENC using Douglas-Peucker algorithm and we arranged the effect of simplified spatial information through CALS method which compensates the defect of basic tree node method.
\end{abstract}

Key words : e-Navagation, IMO, Maritime spatial information, Map generalization, ENC

\section{Introduction}

$\mathrm{e}^{-}$Navigation strategy IMO promotes emphasizes the necessary of means for maximizing efficiency on the use and importance of information for marine transportation services and safe navigation on the entire routes. In the field of shipping mobile telecommunication necessity and demands of various marine spatial informations and high quality communication system are increasing nowadays. However, those systems and the communication networks straggled and they have limited to transmit the various types of high-capacity marine spatial information technically(Jang, Jo, 2005). In the field of land it has been studied about methodology which can improve economics and efficiency in the industry of geographical information through map generalization already. It is expected to need application to ENC(Electronic Navigational Chart) to simplify the marine spatial information.

\section{Map line generalization}

A continuing challenge for automating the cartographic process relates to using data from a digital cartographic database for representation at multiple map scales. The challenge involves feature simplification, and specifically the determination of feature details that must either be retained or omitted for appropriate graphic representation. A study related to map generalization has been conducted by Max Eckert, German cartographer at the very first in 1921 by proposing scientific approach method. Map generalization is the method whereby information is selected and represented on a map in a way that adapts to the scale of the display medium of the map, not necessarily preserving all intricate geographical or other details. Well generalized maps are those that emphasize the most important map elements while still representing the world in the most faithful and recognizable way(Kim, 2004). The level of detail and importance in what is remaining on the map must outweigh the insignificance of items that were generalized, as to preserve the distinguishing characteristics of what makes the map useful and important. The generalization of digital spatial data is done for various reasons including to minimize the amount of data to be processed; to filter out errors and consolidate trends; to produce maps at a variety of scales from the one source; to remove modified features for effective cartographic communication. For example, a small scale map of a coastline will not show every cove that might appear on a large scale map. Smaller scale maps may also omit features completely. It is important to note that data can always be generalized to a smaller scale but cannot be created for larger scales. Generalization of vertor data can be applied to points, lines and polygons and there

\footnotetext{
† Responsible and corresponding author, kohyun@kordi.re.kr 042)866-3694

* osw1@kordi.re.kr 042)866-3692

** pianows@kordi.re.kr 042)866-3690

*** shsuh@kordi.re.kr 042)866-3680

**** cyoun@cnu.ac.kr 042)821-6275
} 
are a number of different algorithms that are used in GIS to handle these processes. Two commonly used generalization operations that can be applied to line data are line simplification and line smoothing. Line simplification algorithms usually remove from the line redundant or unnecessary coordinate pairs based on some geometric criterion, such as distance between points or displacement from a centerline, and they can result in reduced plotting item due to the reduction in the number of $\mathrm{x}, \mathrm{y}$ coordinate pairs; reduced storage space due to the reduction in data by up to $70 \%$ resulting in the decrease in the amount of storage space required(Kim, Lee, 1992). In this study, Douglas-Peucker line simplification algorithm considered as effective algorithm is used to generalize ENC coastline.

Table 1 . The type of generalization algorithms

\begin{tabular}{|c|c|c|}
\hline Object & $\begin{array}{l}\text { Processing } \\
\text { techniques }\end{array}$ & Algorithms \\
\hline \multirow{3}{*}{ Point } & Point to Point & $\begin{array}{l}\text { Cluster } \\
\text { Analysis }\end{array}$ \\
\hline & Point to Line & $\begin{array}{l}\text { Cluster } \\
\text { Analysis }\end{array}$ \\
\hline & $\begin{array}{l}\text { Point to } \\
\text { Polygon }\end{array}$ & $\begin{array}{l}\text { Cluster } \\
\text { Analysis }\end{array}$ \\
\hline \multirow{4}{*}{ Line } & $\begin{array}{l}\text { Independent } \\
\text { Point }\end{array}$ & $\begin{array}{l}\text { Regular } \\
\text { Sampling }\end{array}$ \\
\hline & $\begin{array}{l}\text { Local } \\
\text { Processing }\end{array}$ & $\begin{array}{l}\text { Distance } \\
\text { Calculation } \\
\text { between } \\
\text { Points } \\
\end{array}$ \\
\hline & $\begin{array}{l}\text { Constrained } \\
\text { Condition }\end{array}$ & $\begin{array}{l}\text { Lang } \\
\text { Algorithm }\end{array}$ \\
\hline & $\begin{array}{l}\text { Full coverage } \\
\text { Processing }\end{array}$ & $\begin{array}{l}\text { Douglas-Peucker } \\
\text { Algorithm }\end{array}$ \\
\hline \multirow[b]{2}{*}{ Polyline } & Raster & Zero-Crossing \\
\hline & Vector & $\begin{array}{l}\text { Douglas-Peucker } \\
\text { Algorithm }\end{array}$ \\
\hline
\end{tabular}

\section{Douglas-Peucker Algorithm based on Tree node structure}

The Douglas-Peucker algorithm is an algorithm for reducing the number of points in a curve that is approximated by a series of points by setting up the tolerance band which can decide the level of simplification (Hwang, 1999). The initial form of the algorithm was independently suggested in 1972 by Urs Ramer and 1973 by David Douglas and Thomas Peucker and several others in the following decade. Whereas other line feature generalization algorithms use closeness of vertices as a removal criterion, Douglas-Peucker algorithm uses the closeness of a vertex to an edge segment. This algorithm works from the top down by starting with the single edge joining the first and last vertices of the polyline. Then the other remained vertices are examined to be chosen for closeness to that edge(Douglas, Peucker, 1973). If there are vertices further than an appointed tolerance, then the vertex which is furthest among them can be the simplification vertex. Using recursion, this process is repeated continuously until all vertices of the original polyline are within tolerance of the simplification.
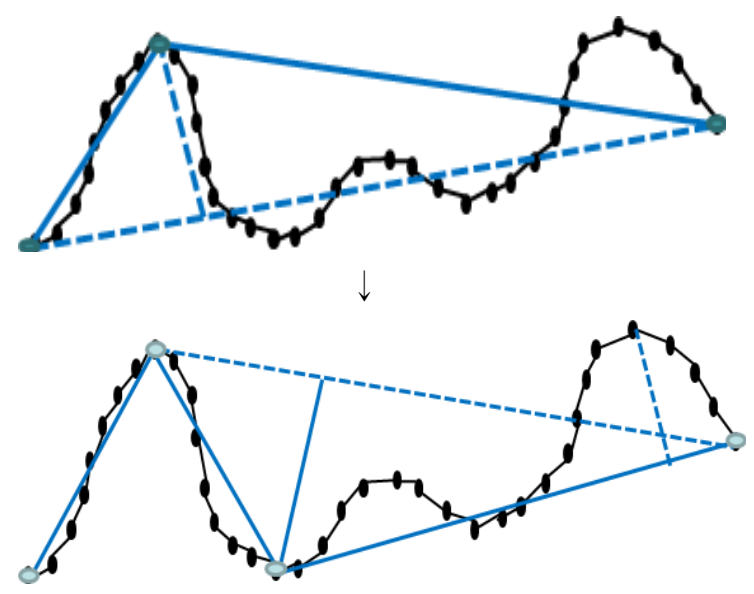

Fig. 1. The process of Douglas-Peucker algorithm

When this iterative algorithm is performed the most important point is to calculate the perpendicular distance. To perform this process the shortest distance between one point and the straight line formula is used. The process of calculating of that formula is that an equation is solved by using each end point first.

$$
y=\frac{-a b x_{1}+a^{2} y_{1}-b c}{a^{2}+b^{2}}
$$

After measuring the $\mathrm{x}, \mathrm{y}$ coordinates of each point on the line,

$$
x=\frac{b^{2} x_{1}-a b y_{1}-a c}{a^{2}+b^{2}}, y=\frac{-a b x_{1}+a^{2} y_{1}-b c}{a^{2}+b^{2}}
$$

the distance between the coordinates of an intersection point and the point calculates.

$$
\frac{\left|a x_{1}+b y_{1}+c\right|}{\sqrt{a^{2}+b^{2}}}
$$


Using (3) formula from above calculating, perpendicular distance can be solved. (Kim, 2004)

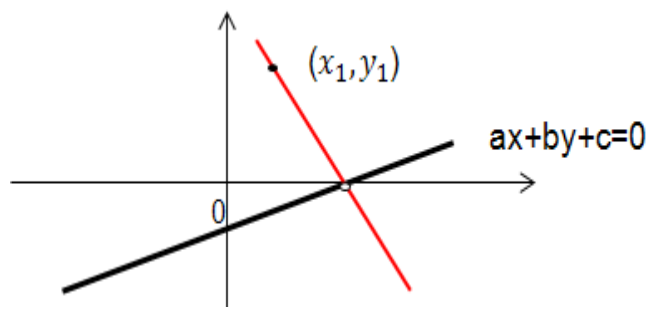

Fig. 2. A mathematical formula describing perpendicular distance

This perpendicular distance can be used to establish binary tree structure, but there is a point to note. The original binary tree formulation rule is to maintain the order of key value of 'left child $<$ parents < right child'. In the case of Douglas-Peucker algorithm, key value is the perpendicular distance. However, if binary tree is established by this perpendicular distance that tree structure is against the binary tree formulation rules. As a result, the index of the binary tree in this study should be established by order of the point which is extracted from the generalization.

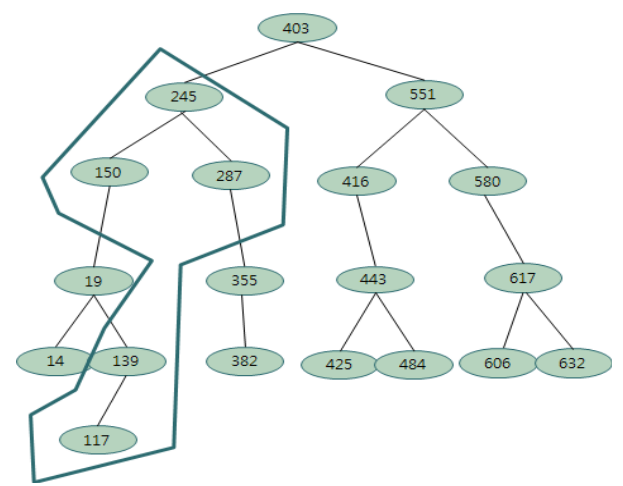

Fig. 3. Basic binary tree index

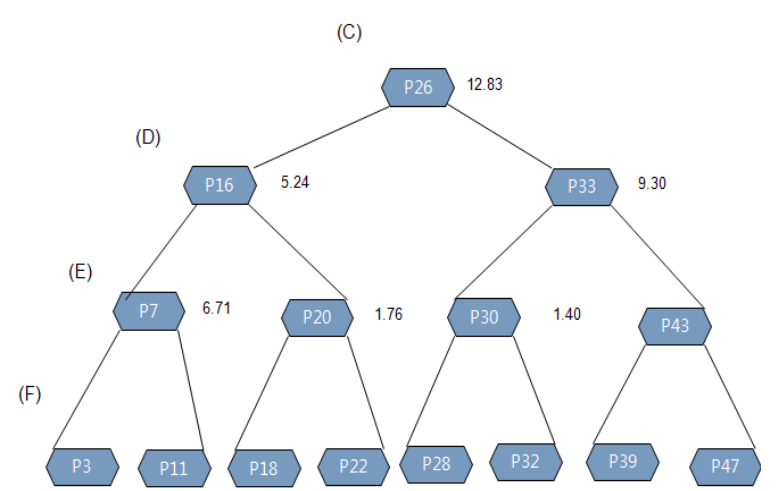

Fig. 4. binary tree index using Douglas-Peucker algorithm
After establishing the binary tree, each perpendicular distance should be compared to the specified actual index value. If index value is smaller than specified one, corresponding perpendicular distance value is removed. For example, if the index value is specified by 6.0 the index which perpendicular distance is more than 6.0 could be extracted. These extracted points only can be generalized points.

(A)

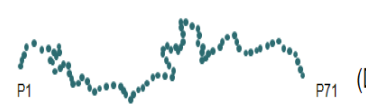
(D)

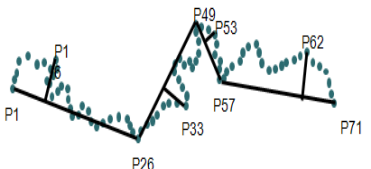

(B)

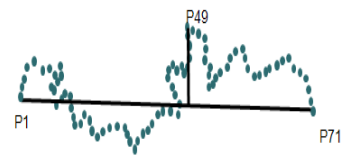

)

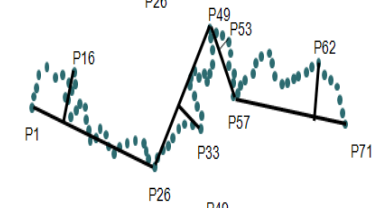

(C)
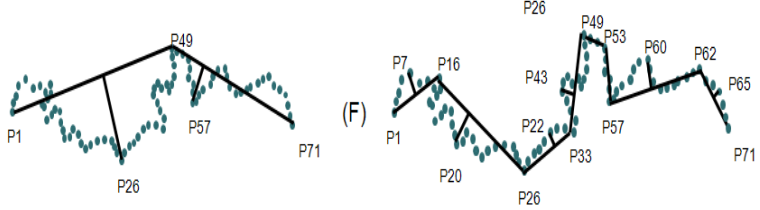

Fig. 5. Douglas-Peucker algorithm based on tree node

However, there is a possibility to have contradiction when simplification is conducted by Douglas-Peucker algorithm using tree structure. This contradiction have a effect to accuracy of the simplification. In the hierarchical tree structure with a set of linked nodes, index value is compared to saved node value to exclude the node which have a value that is the smaller than that index value. However, If the below node value is bigger than above the hierarchical disturbance that have a strong influence on the accuracy comes out. That is why this structure can bring about the contradiction. One of the reason why this error occurs is that Douglas-Peucker algorithm is a full coverage processing which cannot reflect local line feature enough.

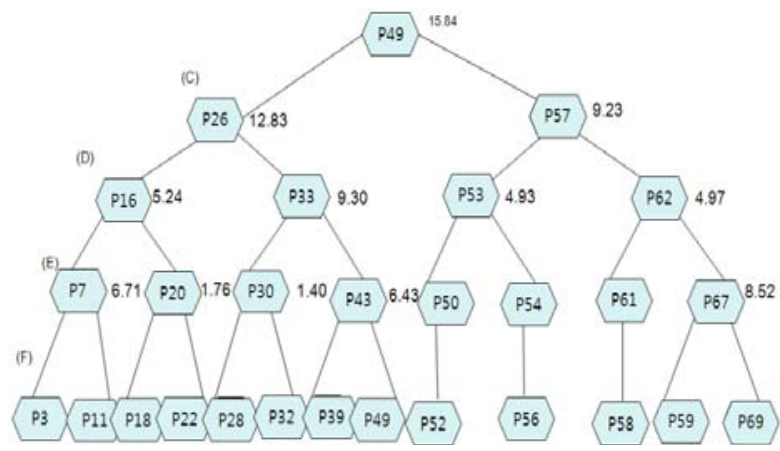

Fig. 6. Tree node hierarchical model 
To overcome this error using Douglas-Peucker algorithm and the advantage of hierarchical tree structure it is need to apply the method reflected in local line feature to tree structure. This is used by CALS(Convex hull Applied Line Simplification) that extracts critical points. In CALS method the points comprising convex hull are found on the all points to get critical points

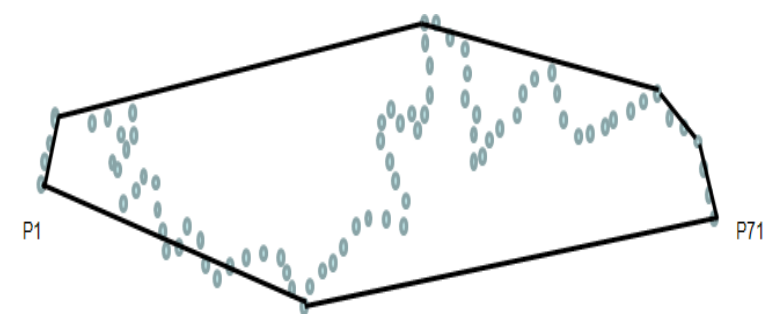

Fig. 7. Establishment of convex hull

The convex hull for a set $\mathrm{X}$ of point in the Euclidean plane is the minimal convex set containing $\mathrm{X}$. For instance, when $\mathrm{X}$ is a bounded subset of the plane, the convex hull may be visualized as the shape formed by a rubber band stretched around X. Algorithms that construct convex hulls of various objects have a broad range of applications in mathematics and computer science. Numerious algorithms are proposed for computing the convex hull of a finite set of points, with various computational complexities. Computing the convex hull means that a non-ambiguous and efficient representation of the required convex shape is constructed. [Hershberger and Snoeyink, 1992] describe an interesting improvement for speeding up the Douglas-Peucker algorithm, making it a worst case $\mathrm{O}(\mathrm{n} \log$ n) time algorithm. In this study, it is referred to the fact that selected points include convex hull in the case of the simplification using Douglas-Peucker algorithm, and then established by the method which calculates simple packing route. There are several algorithms to find convex hull such as package wrapping, Jarvis's March algorithm and Graham algorithm. In this study, Jarvis's March algorithm that is similar to package wrapping, but simpler than that in the angle calculation process is used.

Jarvis's March algorithm is very similar to package wrapping. This is perhaps the most simple-minded algorithm for the convex hull, and yet in some cases it can be very fast. The basic idea of this algorithm is that it starts at some extreme point, which is guaranteed to be on the hull. At each step, each of the points is tested, and the one which makes the largest right-hand turn is found. That point has to be the next one on the hull. Because this process marches around the hull in counter-clockwise order, like a ribbon wrapping itself around the points, this algorithm also called the "gift-wrapping" algorithm.
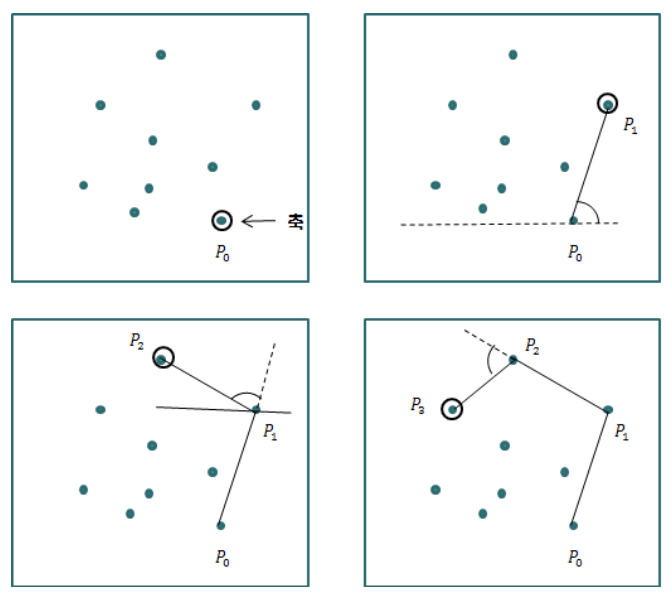

Fig. 8. Package Wrapping

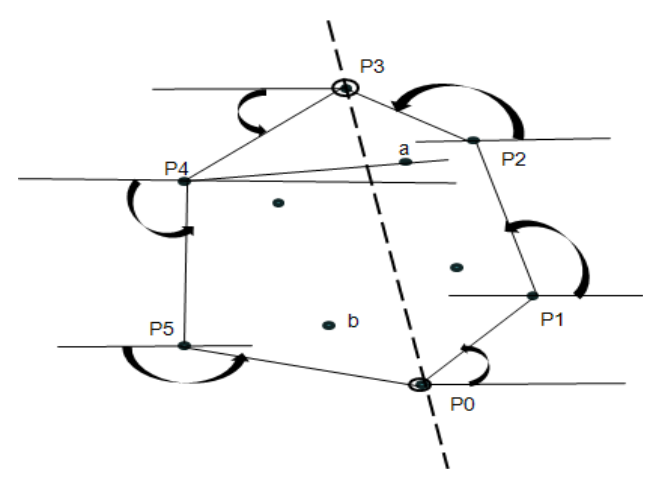

Fig. 9. Jarvis's March Algorithm

The difference between Jarvis's March algorithm and package wrapping is the process finding the set of convex hull points. Jarvis's March algorithm is divided into upward level and downward level. That is because the process that calculates the angle is much simpler than package wrapping. The start point is same as wrapping package which begins at the lowest point of the $\mathrm{y}$ coordinate. However, in this algorithm the highest point of the $\mathrm{y}$ coordinate is also the datum point. Although at the first point the process is conducted by the $x$ coordinate's positive sense when it reaches at another datum point after it changes downward level it takes the angle to negative sense of $\mathrm{x}$ coordinate. If it continues to measure the angle to positive sense at downward level the error would be occurred. As it is shown at Fig. 9, point a becomes the next point to find convex hull point next to the P4. However, this point cannot be the convex hull point. That is why the angle measurement is changed from positive into negative. 
Critical points are extracted from this convex hull, and these points are designed at minimum points of maintenance. After that, simplification is implemented through the hierarchical tree structure. Like this, because critical points are the minimum points of maintenance regardless of the level of simplification local line feature can be reflected when using Douglas-Peucker algorithm. Accuracy can be also improved by reducing the disturbance that can be generated at basic tree structure.

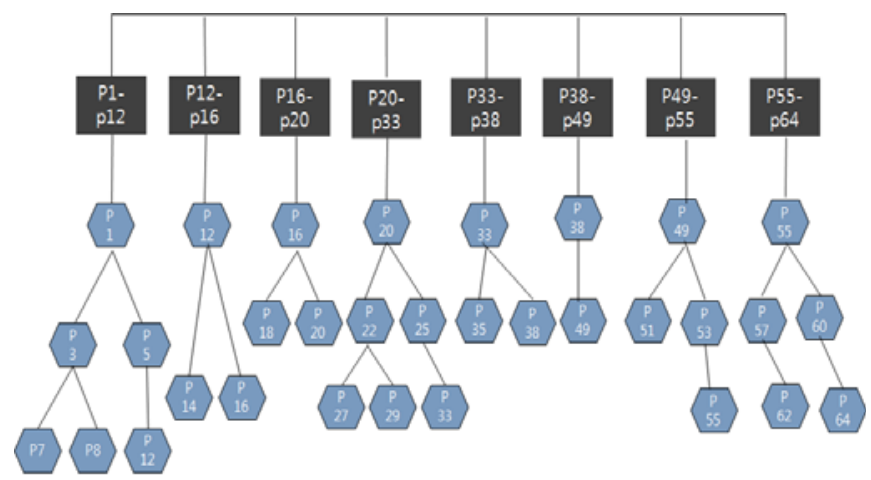

Fig. 10. Data structure applied CALS method

\section{An Application of ENC}

In this study ENC line feature simplification is mapped out by improved CALS method considering local line feature using tree structure generalization method based on Douglas-Peucker algorithm.

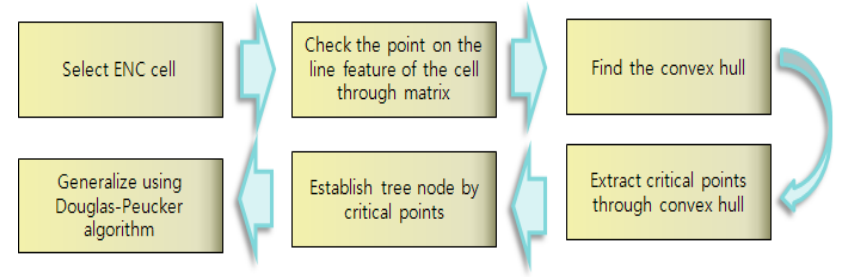

Fig. 11. Process of ENC application by tree data structure with CALS method

An ENC application procedure of CALS method comprises 5 phases. First, the ENC cell containing line feature is selected. In this study we choose KR1G0000.000 file and KR1F0000.000 file. These files' Navigational purpose is overview. Sounding datum is nearly lowest low water, and vertical datum is mean sea level. Next, algorithm establishing convex hull applies to line feature of selected cell, and then extract critical points. After finding these critical points, tree node can be established. Last, generalization is conducted by applying Douglas-Peucker algorithm to ENC cell.

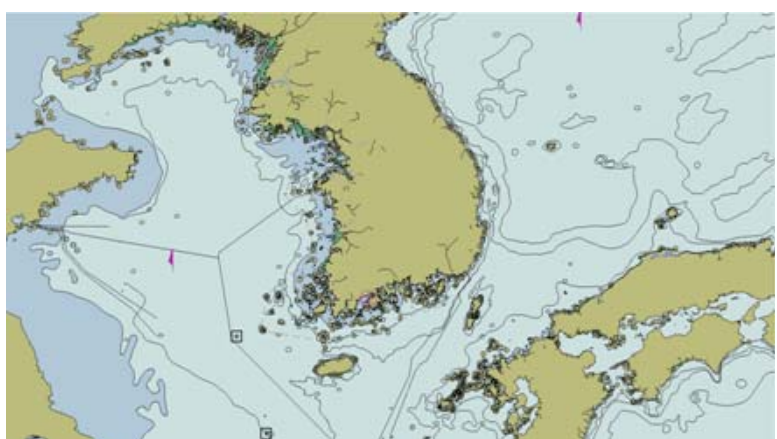

Fig. 12. KR1G0000.000 file and KR1F0000.000 file on the ENC viewer

Fig 12 displays ENC cell generalized through ENC viewer program.

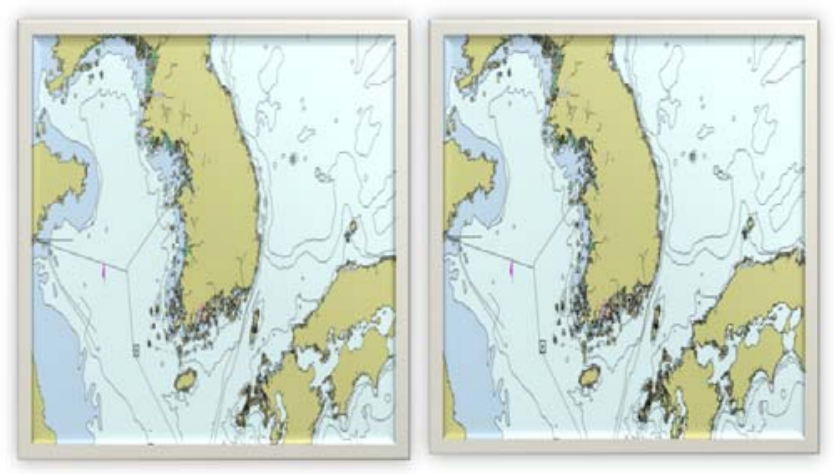

Fig. 13. ENC and ENC applied generalization

From the comparing between original coastline and generalized line it is found that generalized line can be clear and simpler than original one.

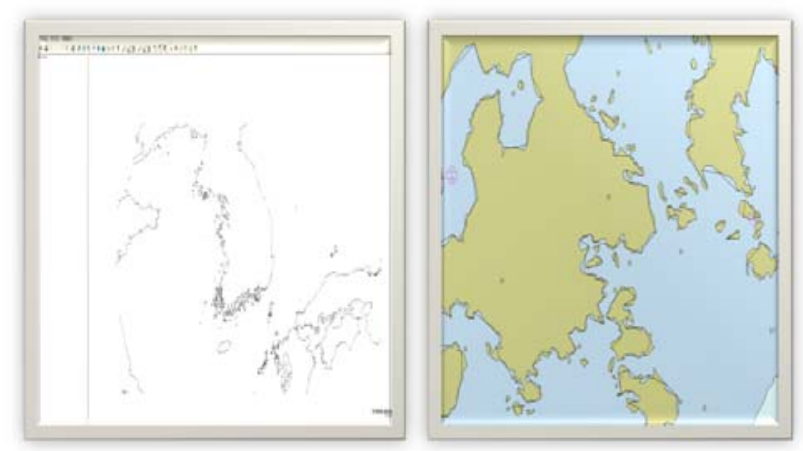

Fig. 14. generalized line shp file and ENC applied generalization enlarged

Left figure is the shape file generalized line feature except for ENC file, and right one is the expanded part of 
the ENC file which is generalized to check details.

In this study ENC coast line is generalized by CALS method. To implement CALS method convex hull algorithm is used. Through this process critical points are extracted and tree node is established. Using tree node structure Douglas-Peucker algorithm is applied to ENC coast line.

\section{Conclusion}

It is considered that the needs of network and information standard on maritime safety that is defined $\mathrm{e}^{-}$Navigation strategy to provide inland and onboard users various informations. However, current maritime communication technology cannot follow the requirements. For this reason, it is required to study that high-capacity maritime safety information can optimize and simplify the line feature. In this study, ENC information is generalized among maritime safety information to simplify the coast line. Although there are a lot of generalization methods Douglas-Peucker algorithm is assessed as one of the most efficient in line feature algorithms. To conduct generalization Douglas-Peucker algorithm is analyzed. Further, hierarchical data structure is used to foster efficiency of simplification process. Generalization accuracy is elevated by using CALS(Convex hull Applied Line Simplification) method rather than basic tree node to consider local line feature. As a result of that, ENC coast line data is simplified it is expected to be possible that data process between inland and onboard is improved more quickly and efficiently.

\section{References}

[1] Hwang, C.S.(1999), “A Study on The Improvement of Douglas-Peucker's Polyline Simplification Algorithm", Journal of Korean Society of Surveying Geodesy Photogrammetry and Cartography, Vol17, No2, pp. $117-128$

[2] Kim, G.R., Lee, H.N.(1992), "A Study on the Evaluation of Simplification Algorithms Based on Map Generalization”, Journal of Korean Society of Surveying Geodesy Photogrammetry and Cartography, Vol10, No2, pp. 63-71.

[3] Jang, D.W., Jo, P.D.(2005), “A Study on Technical Trends for Modernization of the Marine Mobile Communication Systems in International Organizations", Journal of Korea Institute of Information and Communication Engineering, Vol. 14,
No. 2, pp.135-138

[4] Douglas, D. H. and Peucker, T. K.(1973), The Canadian Cartographer, "Algorithms for The Reduction of The Number of Points Required to Represent a Digitized Line or its Caricature”, Vol. 10, No. 2, pp.112-122

[5] Kim, N.S.(2004), “A Cartographic Generalization for Correcting Spatial Errors of Linear Features", Journal of The Korean Association of Geographic Information Studies, Vol7, No1, pp. 39-51.

Received 16 May 2012

Revised 28 June 2012

Accepted 29 June 2012 